systemic inflammation and endothelial dysfunction, which promotes accelerated atherosclerosis ${ }^{2}$

Objectives: Evaluate the frequency of atheromatous plaques in patients with systemic lupus erythematosus.

Methods: Observational, prospective, cross-sectional study. Carotid Doppler was performed on patients with SLE from the external consultation of the rheumatology service from November 2019 to 2020. Inclusion criteria: > 18 years old, diagnosis SLE with the classification criteria ACR 2007, realization of Doppler. Controls: no disease, equated by age and sex. The data was analyzed with SPSS V23.

Results: 116 patients met inclusion criteria, including 116 female controls. Mean sick time was 6.23 years. $14.65 \%$ (17) had atheromaus plates, $29.4 \%$ calcified plates (5). 34.7\% Dyslipidemia (63.1\%) (73), obesity $34.7 \%$ (33), high blood pressure $23.1 \%(22)$, diabetes $3.44 \%(4)$, smokers $0 \%(0)$. The activity rate using SLEDAI showed $68.96 \%$ (80) without activity, $13.79 \%$ (16) low, $11.20 \%$ (13) moderate, $6.03 \%$ (7) high activity. About control group (116), 19.82\% (23) showed atheromatous plates, $39.13 \%$ (9) calcified plates.

Conclusion: Our study shows that less than a quarter of patients have atheromatous plaques in the carotid Doppler. In relation to LES activity, the vast majority are in low activity. We suggest the realization of Carotid Doppler in patients with low activity SLE for evaluation and monitoring of cardiovascular risk. Our study showed that there is no increased risk of atheroma plaque formation in SLE patients, compared to the general population.

REFERENCES:

[1] Hernández Muñiz, Y., Guibert Toledano, Z. and Reyes Llerena, G., 2015 Correlation of $\mathrm{C}$ Reactive Protein Figures and Atherosclerosis In Patients with Systemic Lupus Erythematosus.

[2] Saldarriaga Rivera, L., Ventura Ríos, L., Hernández Díaz, C. and Pineda Villaseñor, C., 2016. Measurement of the thickness of the intimate-half carotid: utility and ultrasound diagnosis of subcline atherosclerosis in rheumatic diseases. Literature review. Rev Col Reum, 23(2), pp.92-101.

[3] Telles, R., Lanna, C., Ferreira, G., Souza, A., Navarro, T. and Ribeiro, A., 2008. Carotid atherosclerotic alterations in systemic lupus erythematosus patients treated at a Brazilian university setting. Lupus, 17(2), pp.105-113.

[4] Nienhuis, H., by Leeuw, K., Bijzet, J., van Doormaal, J., van Roon, A., Smit, A., Graaff, R., Kallenberg, C. and Bijl, M., 2010. Small artery elasticity is decreased in patients with systemic lupus erythematosus without increased intima media thickness. Arthritis Research \& Therapy, 12(5), p.R181.

[5] Frerix et al. Arthritis Research \& Therapy 2014, 16: R54.

[6] Marta, M., Joan T., Stefano B., Chapt 2 - Assessment of Disease Activity in Systemic Lupus Erythematosus, Systemic Lupus Erythematosus, Mosby, 2007.

Disclosure of Interests: None declared

DOI: 10.1136/annrheumdis-2021-eular.2745

\section{AB0326 \\ USEFULNESS OF THE INTIMA-MEDIA THICKNESS INDEX BY CAROTID DOPPLER IN PATIENTS WITH SYSTEMIC LUPUS ERYTHEMATOSUS OF HOSPITAL DOCENTE PADRE BILLINI, DOMINICAN REPUBLIC}

J. Santana Peralta ${ }^{1}$, T. Polanco Mora ${ }^{1}$, A. Cornelio ${ }^{1}$, Y. Cruz ${ }^{1}$, E. Rodriguez Bautista ${ }^{1}$, T. Valdez ${ }^{1}$, R. Muñoz ${ }^{1}$, A. Feriz ${ }^{1} .{ }^{1}$ Hospital Docente Padre Billini, Rheumatology, Santo Domingo, Dominican Republic

Background: Systemic lupus erythematosus (SLE) is a multisistemic autoimmune disease. ${ }^{1}$ There are studies where the increase in the thickness of the intimate/media average in carotid (IMT) is valued, most have shown increase ${ }^{2}$. Cardiovascular disease is one of the leading causes of morbidity, especially due to its precocity, which occurs in women during the fertile potential, is associated with a higher prevalence of cardiovascular disease (CVD), due to accelerated atherosclerosis ${ }^{3,4,5}$. Patients with rheumatic diseases have an increased cardiovascular risk due to systemic inflammation and endothelial dysfunction, which promotes accelerated atherosclerosis ${ }^{2}$ Values below $0.9 \mathrm{~mm}$ of IMT are considered normal.

Objectives: Evaluate the thickness of the intimate/media average in carotid in patients with systemic lupus erythematosus.

Methods: Prospective, Observational, cross-sectional study. Carotid Doppler was performed on patients in the outpatient clinic with a diagnosis of SLE from November 2019 to 2020 of the rheumatology service of the Hospital Docente Padre Billini and healthy controls. Inclusion criteria: > 18 years old, SLE diagnosis with ACR 2007 classification criteria, carotid Doppler, measurement of IMT. Controls without disease, matched by sex and age. The data was analyzed with SPSS V23.
Results: 116 patients with SLE. 95 patients met inclusion criteria;95 healthy controls were included. $97.8 \%$ female. Average disease of 6.23 years. $71.57 \%$ (68) use glucocorticoids, antimalarials 70.52\% (67), 38.94\% (37) mofetil mycophenolate, 20\% (19) methotrexate, $11.57 \%$ (11) rituximab, 5.26\% (5) azathioprine, $1.05 \%$ (1) cyclosporine, $1.05 \%$ (1) cyclophosphamide and $0.86 \%$ (1) tacrolimus. Dyslipidemia $(63.1 \%)(73)$, obesity $34.7 \%(33)$, high blood pressure $23.1 \%(22)$, diabetes $3.44 \%(4)$, smokers $0 \%(0)$. The carotid doppler with SLE showed $17.89 \%$ (17) atheromatous plates, $29.4 \%$ (5) calcified plates, Carotid Doppler in healthy controls showed $20 \%$ (19) atheromatous plates, $36.84 \%$ (7) calcified plates. The activity rate using SLEDA showed $68.96 \%$ (80) without activity, $13.79 \%$ (16) low, $11.20 \%$ (13) moderate, $6.03 \%(7)$ high activity. $78 \%(75)$ patients with SLE had increased IMT mean (SD) $2.15 \mathrm{~mm}$ (0.99). About control $71.57 \%$ (68) had an increase of IMT, mean (SD) $1.27 \mathrm{~mm}$ (1.07) (p-0.046).

Conclusion: Our study found that most patients with SLE had IMT increase. The activity ratio of SLE showed that the vast majority of our patients are in low activity. Alterations in IMT were associated with low SLEDAI and glucocorticoid use. There was no significant difference in the intima-media carotid thickness index with respect to the control group. We suggest the realization of Doppler in patients with SLE despite being in low activity for evaluation and monitoring of cardiovascular risk

\section{REFERENCES:}

[1] Hernández Muñiz, Y, Guibert Toledano, Z. and Reyes Llerena, G., 2015. Correlation of $C$ Reactive Protein Figures and Atherosclerosis in Patients with Systemic Lupus Erythematosus.

[2] Saldarriaga Rivera, L., Ventura Ríos, L., Hernández Díaz, C. and Pineda Villaseñor, C., 2016. Measurement of the thickness of the intimate-half carotid: utility and ultrasound diagnosis of subcline atherosclerosis in rheumatic diseases. Literature review. Rev Col Reum, 23(2), pp.92-101.

[3] Telles, R., Lanna, C., Ferreira, G., Souza, A., Navarro, T. and Ribeiro, A., 2008. Carotid atherosclerotic alterations in systemic lupus erythematosus patients treated at a Brazilian university setting. Lupus, 17(2), pp 105-113.

[4] Nienhuis, H., by Leeuw, K., Bijzet, J., van Doormaal, J., van Roon, A. Smit, A., Graaff, R., Kallenberg, C. and Bijl, M., 2010. Small artery elasticity is decreased in patients with systemic lupus erythematosus without increased intima media thickness. Arthritis Research \& Therapy, 12(5) p.R181.

[5] Frerix et al. Arthritis Research \& Therapy 2014, 16: R54

Disclosure of Interests: None declared

DOI: 10.1136/annrheumdis-2021-eular.2750

\section{AB0327 DRUG-INDUCED LUPUS ERYTHEMATOSUS SECONDARY TO ANTI-TNF-A AGENTS IN PATIENTS WITH SPONDYLOARTHRITIS AND PSORIATIC ARTHRITIS}

A. Martins $^{1}$, D. Santos Oliveira ${ }^{1,2}$, F. R. Martins ${ }^{3}$, M. Rato ${ }^{1}$, F. Oliveira Pinheiro ${ }^{1}$, D. Fonseca ${ }^{4}$, S. Garcia ${ }^{1}$, B. M. Fernandes ${ }^{1}$, S. Pimenta ${ }^{1,5}$, M. Bernardes ${ }^{1,5}$, L. Costa ${ }^{1}{ }^{1}$ Centro Hospitalar Universitário São João, Rheumatology, Porto, Portugal; ${ }^{2}$ Faculdade de Medicina da Universidade do Porto - FMUP, Center for Health Technology and Services Research (CINTESIS), Porto, Portugal; ${ }^{3}$ University Hospital Center of Algarve, Rheumatology, Faro, Portugal; ${ }^{4}$ Centro Hospitalar Vila Nova de Gaia / Espinho - Unit 1, Rheumatology, Vila Nova de Gaia, Portugal; ${ }^{5}$ Faculdade de Medicina da Universidade do Porto - FMUP Rheumatology, Porto, Portugal

Background: Induction of autoantibodies is frequently observed in patients treated with TNF- $\alpha$ antagonist and the possible development of drug-induced lupus erythematosus (DILE) remains a matter of concern. The prevalence of DILE secondary to anti-TNF- $\alpha$ therapy is estimated around $0.5-1 \%$ and clinica features include arthritis/arthralgia, rash, serositis, fever, myalgias, cytopenias, among others. According to the literature, DILE secondary to anti-TNF- $\alpha$ agents differs in several ways from the clinical and laboratory findings typically associated with classic DILE.

Objectives: To estimate the incidence of induction of antinuclear antibodies (ANA) and DILE in a monocentric cohort of patients with spondyloarthritis and psoriatic arthritis treated with anti-TNF- $\alpha$ agents. To describe the clinical and laboratorial features and outcomes of patients with DILE.

Methods: We performed a retrospective analysis of patients with spondyloarthritis and psoriatic arthritis treated with anti-TNF- $\alpha$ agents, from our University Hospital, who have been registered on the Portuguese Rheumatic Diseases Register (Reuma.pt) between July 2001 and December 2020. Patients with 
positive ANA (titer > 1/100) before the anti-TNF- $\alpha$ therapy were excluded. Because specific criteria for the diagnosis of DILE have not been established, we considered the diagnosis in case of a temporal relationship between clinical manifestations and anti-TNF- $\alpha$ treatment and fulfillment of ACR/EULAR 2019 classification criteria for SLE. In patients with DILE, clinical features, laboratory findings, systemic therapies and outcome after discontinuation of medication were collected from reuma.pt and medical records. For the clinical and demographic predictors, continuous variables were analyzed using a two-sided t-test and categorical variables using a Fisher's exact test. P-value $<0.05$ was considered statistically significant.

Results: In the spondyloarthritis group, 290 patients were included (44.8\% females, mean age at diagnosis of $33.3 \pm 11.5$ years and mean disease duration of $15.1 \pm 10.4$ years) and in the psoriatic arthritis group, 116 patients were included $(50.0 \%$ females, mean age at diagnosis of $40.1 \pm 11.0$ years and mean disease duration of $13.1 \pm 6.8$ years). In our study, we observed high serology conversion rates (positive ANA in $67.9 \%$ and $58.6 \%$ of patients with Spondyloarthritis and Psoriatic Arthritis, respectively), with similar conversion rates between different anti-TNF drugs. Three patients with spondyloarthritis (1.0\%) and 1 patient with psoriatic arthritis $(0.9 \%)$ developed DILE. Etanercept was the causative agent in 2 cases, infliximab and adalimumab in 1 case, each. Peripheral arthritis (new onset or abrupt worsening) occurred in 2 patients, serositis in 1 patient, constitutional symptoms in 2 patients, subnephrotic proteinuria in 1 patient, lymphopenia in 2 patients and hypocomplementemia in 1 patient. Specific treatment was prescribed to the 4 patients (oral corticosteroids) and they achieved complete recovery. After anti-TNF- $\alpha$ treatment interruption, no patient had recurrent disease. We observed that patients with DILE had a significantly longer disease duration ( $>8.4$ years; $p=0.04$ ) and a significantly longer duration of therapy with anti-TNF ( $>4.0$ years; $p=0.04$ ) when compared to patients without DILE.

Conclusion: Despite the frequent induction of autoantibodies, the development of DILE secondary to anti-TNF- $\alpha$ agents is rare. Our study demonstrates an incidence rate similar to other studies reported before. The clinical and laboratorial characteristics of our patients with DILE attributable to anti-TNF- $\alpha$ agents differ significantly from DILE due to more traditional agents, as is described in literature. Overall, patients in this study had mild disease that improved after therapy discontinuation, without recurrence of the disease. It seems that a longer disease duration and a longer period under anti-TNF- $\alpha$ therapy may increase the risk of DILE development.

Disclosure of Interests: None declared

DOI: 10.1136/annrheumdis-2021-eular.2953

\begin{tabular}{l|l}
\hline AB0328 & CLINICAL AND SEROLOGICAL DIFFERENCES \\
BETWEEN PRIMARY SJOGREN'S SYNDROME \\
PHENOTYPES
\end{tabular}

$\underline{\text { X. E. Larco Rojas }}{ }^{1}$, E. Diez Alvarez ${ }^{1}$, C. Moriano ${ }^{1}$, A. López Robles ${ }^{1}$, T. Pérez Sandoval ${ }^{1} .{ }^{1}$ Complejo Asistencial Universitario de León, Rheumatology, León, Spain

Background: Primary Sjogren's Syndrome (PSS) is an autoimmune and lymphoproliferative disease with a heterogeneous presentation. It has been postulated that there may be different phenotypes, in some cases presenting a more aggressive disease with systemic manifestations and a higher risk of developing complications. This phenotype has been associated with a higher autoimmune load and an earlier age of presentation. Furthermore, the presence of anti La + has been related to an increased risk of developing Lymphoma.

Objectives: To describe the phenotypic characteristics of seronegative PSS in a sample of patients from our practice. To compare the clinical and laboratory characteristics between patients with $\mathrm{Ro}+/ \mathrm{La}+$ and $\mathrm{Ro}+$ / La- antibodies. To Analyze if there are differences in patients diagnosed at an early age, compared to a later age.

Methods: Clinical and serological characteristics of patients with the diagnosis of PSS were collected from the Rheumatology database of León`s Hospital between 2014-2020. All patients who met the ACR / EULAR 2016 criteria were classified as seronegative Sjogren.

In the group of patients with positive autoimmunity, anti-Ro + / La + were compared with the anti-Ro $+/$ La- patients and by age, stratifying them into the following groups: $0-49 ; 50-69$ and $>70$ years. The clinical variables analyzed were: glandular inflammation, Raynaud's phenomenon (RP), pulmonary and neurological involvement, presence of Lymphoma and other tumours. The serological variables were: positivity of ANA, Rheumatoid Factor (RF), hypocomplementemia, hypergammaglobulinemia and B2 microglobulin.

Results: 72 patients were analysed, 9 were excluded because didn't meet the criteria. Of the remaining: $90,4 \%$ were women, with a mean age of $58,7+/-15,8$ years, $12,6 \%$ (8) were seronegative. In the seronegative group $25 \%$ presented lung involvement (Lymphoid Interstitial Pneumonia), 50\% presented with glandular inflammation and only one patient had RP. As complications 1 patient presented Lymphoma and 1 Breast Carcinoma.

$58,7 \%$ (37) Ro + / La + and 28,5\% (18) Ro + / La- patients were identified no statistically significant differences were found between the two groups when comparing: glandular inflammation ( $8 / 37$ vs $2 / 18, p=>0.05) R P(9 / 37$ vs $4 / 18, p=>0.05)$, pulmonary involvement $(5 / 37$ vs. $6 / 18, p=>0.05)$, neurological involvement (2/37 vs. $1 / 18, p=>0.05)$, presence of Lymphoma (2/37 vs. $0 / 18, p=>0,05)$, other tumours (2/37 vs $3 / 18, p=>0.05)$, ANA positivity ( $36 / 37$ vs $16 / 18, p=>0,05)$, Hypocomplementemia (4/37 vs $3 / 18, p=>0.05$ ) and Hypergammaglobulinemia (20/37 vs $10 / 18, p=>0.05)$. But a higher frequency of positive RF linked to anti La positivity $(29 / 37$ vs $6 / 18 p=0.002)$ was found.

When comparing by age groups, the association between $\mathrm{RF}+$ and $\mathrm{La}+$ remained in the group of $50-69$ years $(15 / 18$ vs $3 / 18, p=0.002)$ while in the other age groups there were no statistically significant differences. We also observed an increasing trend of the levels of B2microglobulin in La+ patients and later age $(p=0,04)$

Conclusion: The presence of anti $\mathrm{La}+$ seems to be associated with other components of autoimmunity such as RF in patients with PSS, although this study did not show a relation with a higher frequency of complications or systemic disease. Also, the presence of La+ at older ages was associated with higher levels of B2 microglobulin. We didn't find differences with the other described markers of $B$ cell reactivation. Findings differ from those found in the literature, which may be largely due to sample size.

\section{REFERENCES:}

[1] Quartuccio L., Baldini C., Bartoloni E., et al. Anti-SSA/SSB-negative Sjogren's syndrome shows a lower prevalence of lymphoproliferative manifestations, and a lower risk of lymphoma evolution. Autoimmunity Reviews 14 (2015) 1019-1022.

[2] Quartuccio L, Isola M, Baldini C, Priori R, Bartoloni Bocci E, Carubbi F, et al. Biomarkers of lymphoma in Sjögren's syndrome and evaluation of the lymphoma risk in prelymphomatous conditions: results of a multicenter study. J Autoimmun 2014; 51:75-80.

Disclosure of Interests: None declared

DOI: 10.1136/annrheumdis-2021-eular.2959

\section{AB0329 \\ HIGHER PREVALENCE OF ECHOCARDIOGRAPHIC ABNORMALITIES IN PATIENTS WITH SYSTEMIC LUPUS ERYTHEMATOSUS}

N. Guajardo-Jauregui ${ }^{1}$, J. R. Azpiri-López ${ }^{2}$, D. Á. Galarza-Delgado ${ }^{1}$, I. J. Colunga-Pedraza', R. A. Reyna-de la Garza ${ }^{3}$, S. Lugo-Perez ${ }^{2}$, A B. Rodriguez-Romero ${ }^{1}$, A. Cárdenas ${ }^{1}$, H. Azpiri-Diaz², O. A. Cepeda-Ayala ${ }^{2}$. ${ }^{1}$ Hospital Universitario “Dr. José Eleuterio González”, Universidad Autónoma de Nuevo León, Rheumatology, Monterrey, Mexico; ${ }^{2}$ Hospital Universitario “Dr. José Eleuterio González", Universidad Autónoma de Nuevo León, Cardiology, Monterrey, Mexico; ${ }^{3}$ Hospital Universitario "Dr. José Eleuterio González”, Universidad Autónoma de Nuevo León, Internal Medicine, Monterrey, Mexico

Background: Systemic lupus erythematosus (SLE) is a chronic, inflammatory, and autoimmune disease that damages vital organs such as the heart. Patients with SLE have a higher risk of developing a cardiovascular (CV) disease than the general population (1).

Objectives: The aim of this study was to compare the echocardiographic findings in patients with SLE and controls.

Methods: This was a cross-sectional, observational, and comparative study. A total of 38 patients with SLE according to the 2019 EULAR/ACR classification criteria, $\geq 18$ years old and 38 matched controls by age $( \pm 5$ years) and gender, were recruited for this study. Exclusion criteria were a poor echocardiographic window, patients with a previous $\mathrm{CV}$ event, such as myocardia infarction, cerebrovascular event or peripheral arterial disease, and pregnant women. A transthoracic echocardiogram, including speckle tracking technique, 